\title{
Desiccation cracking of soils
}

\author{
H. Peron* - L. Laloui* - T. Hueckel** - L.B. Hu** \\ * Ecole Polytechnique Fédérale de Lausanne, EPFL \\ Laboratory of Soil Mechanics, Station 18 \\ CH-1015 Lausanne, Switzerland \\ herve.peron@epfl.ch \\ ** Duke University, Department of Civil and Environmental Engineering \\ Durham, NC 27708, U.S.A.
}

\begin{abstract}
The scope of this paper is to present the global mechanisms of soil desiccation, including drying shrinkage and cracking. The paper first reviews the basic processes that are beneath the word "desiccation". Then the results of an experimental study of desiccation are presented, in which strains, suction, water content, degree of saturation and crack geometry are investigated. The results show that cracking initiates close to the onset of de-saturation. Insights into the micro-scale are proposed to explain this observation. A scenario for the processes leading to crack initiation is further established in terms of the macroscopic variables: an assessment of the stress building up is proposed, until a critical point at which the tensile strength is met. Desiccation crack pattern formation is finally discussed.

RÉSUMÉ. Le but de cet article est de présenter l'ensemble des mécanismes de la dessiccation des sols, comprenant le retrait de séchage et la fissuration. La nature de ces derniers processus dans les sols est tout d'abord définie. Les résultats d'une étude expérimentale de la dessiccation (qui consiste en une investigation de l'évolution des déformations, de la succion, de la teneur en eau, du degré de saturation et de la géométrie des fissures) sont ensuite exposés. Les résultats montrent que la fissuration commence à un moment proche du début de la désaturation et de la valeur d'entrée d'air. Cette observation est interprétée à l'échelle microscopique. Un scénario pour les processus menant à l'initiation de la fissuration est établi en termes de variables macroscopiques: une évaluation du développement des contraintes jusqu'à un critère macroscopique d'initiation des fissures est proposée. La formation des formes de fissures est finalement discutée.

KEYWORDS: drying shrinkage, cracking, degree of saturation, air entry value, stresses.

MOTS-CLÉS : retrait de séchage, fissuration, degree de saturation, valeur d'entrée d'air, contraintes.
\end{abstract}


870 European Journal of Civil and Environmental Engineering. Volume $13-\mathrm{n}^{\circ} 7$ $8 / 2009$

\section{Introduction}

Drying of soils and the ensuing cracking are a crucial issue in geo-environmental engineering. Drying fractures strongly affect permeability and may compromise the integrity of structures, such as clay buffers for nuclear waste isolation. In addition, cracking is the cause of substantial damage in foundation-supported structures. Compressibility increases substantially while the rate of consolidation decreases with the appearance of desiccation cracks (Morris et al. 1992). Cracks are also a possible precursor for inception of a failure surface at the top of dams and embankments. Assessment of the potential for such damage is difficult, as the mechanisms and principal variables in the process are not fully understood, despite decades of research and substantial progress, e.g., Corte and Higashi (1960), AbuHejleh (1993), Konrad and Ayad (1997).

The primary scope of this paper is to present the global mechanisms of desiccation, including shrinkage and cracking. For this purpose, experimental evidence as well as the substantial advances made in recent years in understanding the hydro-mechanics of unsaturated soils are used. The entire set of processes that lead to cracking and that stem from drying shrinkage is addressed.

The paper first reviews the basic processes that are beneath the word "desiccation". Then the results of an experimental study of desiccation are presented, in which strains, suction, water content, degree of saturation and crack geometry are investigated. The conditions of cracking initiation are discussed, especially with respect to the degree of saturation and suction. Insights into the micro-scale are proposed, bearing in mind that the useful variables are those at the macro-scale. A scenario for the processes leading to crack initiation is established in terms of the macroscopic variables. Desiccation crack pattern formation is finally discussed.

\section{Physical processes involved by desiccation cracking of soils}

\subsection{Drying}

Drying results from an initial thermodynamic imbalance between the soil moisture and its surroundings, which causes evaporation and a transfer of fluids within the soil. In the general case, the fluid movement is accomplished through both liquid and gaseous phases. According to equilibrium thermodynamics laws, the phase change between pore liquid and vapour occurs instantaneously at the interface between the phases, so that the specific vapour and liquid Gibbs potentials remain equal (Coussy et al. 1998, Mainguy et al. 2001). This generates liquid pressure decay (i.e. suction increase) in this zone according to Kelvin's law, and at the same time, a gradient of suction within the body. The liquid movement is due to the spatial differences in liquid head that then arise. External pressures, high 
temperatures and shrinkage deformations are also responsible for additional pore fluid pressure generation and subsequent fluid movements.

We define the velocities of the gas, liquid and solid, respectively, by $\mathbf{v}_{\mathbf{g}}, \mathbf{v}_{\mathbf{l}}$ and $\mathbf{v}_{\mathbf{s}}$. The Darcy's law then relates the liquid/solid relative velocity to the gradient of the water head. The Darcean flux of liquid $\mathbf{q}_{\mathbf{l}}\left[\mathrm{M} \cdot \mathrm{L}^{-2} \cdot \mathrm{T}^{-1}\right]$ is given by:

$$
\mathbf{q}_{\mathbf{1}}=\rho_{l} n S_{r}\left(\mathbf{v}_{\mathbf{l}}-\mathbf{v}_{\mathbf{s}}\right)=-(k / g)\left(-\nabla p_{l}+\rho_{l} \mathbf{g}\right)
$$

where $n$ is the porosity, $S_{r}$ is the degree of saturation, $k$ is the hydraulic conductivity (permeability to liquid) of the medium and $\mathbf{g}$ is the gravity vector, $\rho_{l}$ and $p_{l}$ are the intrinsic density and pressure of the liquid, respectively.

When a continuous gaseous phase is present within the body, the gaseous phase movement can also be described by the Darcy's law, as the consequence of a gradient of gas (fluid) head.

One should also consider transport of vapour associated with evaporation phenomenon. Even in the absence of a gas pressure gradient, vapour diffusion due to a difference in vapour concentration (or mole fraction) occurs and is expressed according to Fick's law, whose applicability to a gaseous mixture has been proven with the kinetic theory of gases. Vapour and dry air are here assumed to form an ideal mixture. Fick's law can be expressed as:

$$
\mathbf{q}_{\mathbf{v}}^{\mathbf{d}}=\rho_{v} n\left(1-S_{r}\right)\left(\mathbf{v}_{\mathbf{v}}-\mathbf{v}_{\mathbf{g}}\right)=-\rho_{g} \mathbf{D}_{\mathbf{v}} \nabla\left(\rho_{v} / \rho_{g}\right)
$$

where $\mathbf{q}_{v}^{d}$ is the diffusive vapour flux with respect to the gaseous mixture, $\mathbf{v}_{\mathbf{v}}$ is the velocity of the vapour and $\rho_{v}$ its density, $\rho_{g}$ is the density of the gaseous mixture, and $\mathbf{D}_{\mathbf{v}}$ is a molecular diffusion tensor representative of vapour diffusion through dry air inside the porous medium. $\mathbf{D}_{\mathbf{v}}$ is different from the molecular diffusion coefficient in a purely gaseous medium as it takes into account the tortuosity of the medium (Philip and de Vries 1957).

In certain situations, other phenomena can take place. Effusion of vapour (Knudsen model) occurs when the mean free path of the vapour molecule is on the same order as the diameter of the pores. Stefan's flux can occur when intensive vapour production creates a significant vapour pressure gradient (Kowalski 2003). The liquid phase may contain dissolved air. In this case, one would have to consider air dissolution into the liquid phase, which is governed by Henry's law, plus subsequent transport of the dissolved air into the liquid, which is mainly governed by Fick's law.

Drying is intrinsically a non-adiabatic process, since it requires energy (needed for evaporation). Under non-isothermal conditions, additional mechanisms of moisture transfer have to be considered during drying. The Soret effect (or thermodiffusion) refers to an additional diffusion-like process of vapour in dry air, 
872 European Journal of Civil and Environmental Engineering. Volume $13-\mathrm{n}^{\circ} 7$ $8 / 2009$

occurring when a temperature gradient exists (Platten 2006). The evaporationcondensation mechanism might also act: liquid particles evaporate faster from the meniscus surface located in the warmer zone than they do from the surface located in the cooler zone. Then, capillary uplift of the liquid bridge or liquid pocket takes place towards the warmer zone because of the difference of surface tensions between menisci (Kowalski 2003).

If the heat needed for evaporation (i.e. the latent heat of evaporation) is only supplied by the soil itself, a straightforward calculation (also carried out by Baggio et al. 1997), tells us that for a saturated clay (liquid is water) having a specific heat of $1.3 \mathrm{~kJ} \cdot \mathrm{kg}^{-1} \cdot \mathrm{K}^{-1}$ and a porosity of 0.3 , the temperature decrease would be about $346 \mathrm{~K}$. However, in general, the heat source is the gas (air) surrounding where the soil dries. In this situation, isothermal drying can be assumed if the phase change rate is limited by the mass transfer, and is therefore not controlled by the heat transfer. This situation is encountered in most of the soils (Peron 2008).

\subsection{Shrinkage}

At the micro-scale it may be considered that the basic process behind the shrinkage of a soil is a decrease in liquid pressure and more generally an increase in suction $s$, caused by the evaporation at the location of interphase menisci, and which acts as an attractive force between the components of the matrix. In the initial, saturated stage of the process the menisci are located at the external boundary of the soil body. At the macro-scale, this translates into application of suction at the boundary and a resulting increase of effective stress compression throughout the soil the matrix and the ensuing sample shrinking. For fine soils with no swelling minerals, and following Mitchell and Soga (2005), it is reasonable to assume that the mechanisms related to the adsorbed water are not prevalent (i.e. capillary processes predominate), at least for a large range of water content and associated shrinkage strains.

Shrinkage strain then can be seen as the consequence of an effective stress increase. At the macro-scale the effective stress embeds the contributions of externally applied stresses and internal pore fluid pressures. The Bishop's generalized effective stress is a standard and broadly recognized effective stress for partially saturated soils (Nuth and Laloui 2008), whose component $\sigma_{i j}^{\prime}$ is expressed as follows:

$$
\sigma_{i j}^{\prime}=\sigma_{i j}^{n e t}+S_{r} s \delta_{i j}
$$

where $\sigma_{i j}^{\text {net }}$ is the net stress component (difference between the total stress component and the air pressure), s the matric suction and $\delta_{i j}$ the Kronecker symbol. 


\subsection{Cracking}

Desiccation macro-cracks are likely to occur if the drying shrinkage is constrained (Corte and Higashi 1960). Typically, these constraints can arise from different causes (Hueckel 1992): (i) a frictional or any other traction or displacement boundary conditions, (ii) any eigen-stress concentrations within the soil sample, and (iii) intrinsic soil inhomogeneity factors, such as soil texture and soil structure. In the field, cause (i) can arise from any restraining structure, and cause (ii) from soil moisture gradients, which do not respect the strain compatibility conditions (see e.g., Kowalski 2003). In this paper, causes (i) and (ii) are focused on. Irrespective of soil internal structure, any soil element that is allowed to dry and is not constrained in its movement by adjacent elements or boundary conditions, would not crack.

In early studies, stress generation and subsequent cracking was related to the change in the rheological properties of soil during drying. Because of the liquid loss, soil loses its ability to relieve the tensile forces generated by the mechanisms explained above. An additional build-up of stress arises, which is finally relieved by tensile failure and cracking (Lachenbruch 1961). When the shrinkage deformations at a macroscale are expressed through a phenomenological approach, i.e. directly relating the development of shrinkage deformations to a non-mechanical convenient variable such as water content or suction, it is convenient to split the total strain increment $d \varepsilon_{i j}$ into a mechanical strain increment $d \varepsilon_{i j}^{m}$ and a shrinkage strain increment $d \varepsilon_{i j}^{h}$ as follows:

$$
d \varepsilon_{i j}=d \varepsilon_{i j}^{m}+d \varepsilon_{i j}^{h}
$$

In this way, desiccation-induced stress could be dealt with using an approach that is analogous to the one used for thermal stress computation. Many studies aimed at explaining the origin of tensile stress generation during desiccation eventually use such an approach (Kowalski 2003). In reaction to any restraint in its shrinkage deformation, a total stress increment $d \sigma_{i j}$ arises in the considered element of soil. Such a stress increment could be expressed by:

$$
d \sigma_{i j}=D_{i j k l} d \varepsilon_{k l}^{m}=D_{i j k l}\left(d \varepsilon_{k l}-d \varepsilon_{k l}^{h}\right)
$$

where $D_{i j k l}$ is the stiffness matrix component.

Experimental evidence clearly shows that desiccation cracking mainly occurs in mode I (i.e. opening mode), indicating that cracking is the result of soil tensile strength mobilization, as commonly acknowledged. Prediction of desiccation cracking thus entails determination of tensile strength.

Most of the existing yield and failure criteria for soils relate to compression behaviour involving shear resistance. This is not surprising given the low value of 
874 European Journal of Civil and Environmental Engineering. Volume $13-\mathrm{n}^{\circ} 7$ $8 / 2009$

tensile strength usually observed (Mitchell and Soga 2005). However, in order to effectively account for desiccation cracking, a rigorous tensile failure criterion must be addressed. In this sense, the Griffith's criterion deserves consideration. Griffith (1924), working on elastic materials, considered the numerous minute cracks and defects in the materials that were "naturally" present at all times and derived the value of the minor principal stress at failure, defined as the tensile strength. Griffith's criterion is routinely used in rock mechanics to interpret tensile failure. In soil mechanics, it has also been invoked to interpret several traction test results (Bishop and Garga 1969). Frydman (1967) and Morris et al. (1992) note that microcracks in the sense defined by Griffith can be identified within intergranular voids as well.

As soil undergoes drying, it exhibits suction change, which, in turn, causes the inter-particle force to increase. An apparent cohesion term is thus created, and consequently, a tensile strength term. There is thus a coupling between suction and tensile strength, which was recognised early in the study of this field. Some authors, e.g. Schubert (1982) and Kim and Hwang (2003), have attempted to directly relate tensile strength to the normal inter-particle force, calculated from micro-scale considerations. Considering the Griffith theory of tensile failure, it is believed that such approaches give only a partial view of the real mechanisms related to macroscopic tensile failure and the way that tensile strength evolves during drying. These mechanisms remain poorly understood.

Morris et al. (1992) suggest a criterion for cracking initiation based on a modification of the Mohr-Coulomb criterion in the tensile range. It is assumed that the soil experiences tensile strength and apparent cohesion (denoted $c_{a p p}$ ) increases, related to the increase of suction. An arbitrary value of a coefficient $\alpha_{t}$ is set such that $\sigma_{t}=\alpha_{t} c_{a p p} \cot \phi$, where $\sigma_{t}$ is the absolute value of tensile strength and $\phi$ the friction angle. The authors suggest that $\sigma_{t}=c_{a p p} / 2$. One can realize that such a value is close to a "corrected" tensile strength deduced from Mohr-Coulomb criterion, for which tensile stresses normal to the shearing plane are not allowed: $\sigma_{t}$ $=c_{a p p} \cos \phi /(1+\sin \phi)$, for usual friction angles (Peron 2008).

Still adopting a total (or net) stress interpretation of the processes leading to cracking, Snyder and Miller (1985) combined an expression of intergranular stress for unsaturated soil with notions issued from Griffith's theory of fracture of flawed elastic solids. An expression for the tensile strength is derived:

$$
\sigma_{t}=\left(\chi / f_{\text {flaw }}\right) s
$$

$f_{\text {flaw }}$ accounts for the shape and the size of the so-called flaw, $\chi$ depends on the degree of saturation. The ratio $\chi / f_{\text {flaw }}$ decreases with saturation degree; it is almost 1 when pores are small and can reach very low values if pores are big. For a saturated soil, $\sigma_{t}=s$. In the case of uniformly packed particles, (no flaws), $f_{\text {flaw }}=1$, and $\sigma_{t}=\chi s$. The criterion expresses the fact that tensile strength depends on the 
degree of saturation and on suction, and is also related to the most critical flaw in the Griffith sense. A large air-filled pore can play the role of crack inceptor.

\section{Experimental characterization of the process and its controlling variables}

It is intended here to quantify the processes of drying shrinkage leading to desiccation cracking in soils, by establishing experimental relationships between drying and cracking on the one hand and physical variables at the macroscale on the other hand. The variables investigated are water content, suction, saturation degree, strains, stresses, and crack geometry. Konrad and Ayad (1997) performed field desiccation experiments on intact clay; Miller et al. (1998) carried out a small scale desiccation test on landfill liners. Both studies mainly focused on suction evolution during drying and crack pattern geometry, without addressing shrinkage mechanisms, and mechanical conditions of crack initiation. Kleppe and Olson (1985), Daniel and Wu (1993) and Albrecht and Benson (2001) conducted desiccation tests on compacted soils used for clay liners and covers. Their main aim was to estimate the hydraulic conductivity decay of such cracked soil specimens. Only a few empirical relationships between cracking potential, shrinkage strains and compaction conditions have been proposed.

A paramount question that arises during an investigation of desiccation in an initially saturated soil is whether cracking occurs when the soil is still saturated. Related to this topic, cavitation is invocated as a fundamental mechanism of cracking initiation in material such as gels (Scherer 1990), but this requires clarifications when dealing with soils. Some insight into the relationship between cracking and pore water cavitation onset is provided by Hu et al. (2008). Finally, it has been repeatedly noted that the desiccation crack patterns show remarkable periodicity, both in nature and in the laboratory. Their quantification remains elusive for soils.

\subsection{Desiccation tests}

\subsubsection{Desiccation tests on bars}

Clayey silt from Bioley (Switzerland) was prepared as a saturated slurry (gravimetric water content $w=49 \%$ ), and shaped in rectangular bars (length 300 $\mathrm{mm}$, width $50 \mathrm{~mm}$, height $12 \mathrm{~mm}$ ). Details on experiments can be found in Peron et al. (2009) and in $\mathrm{Hu}$ et al. (2006). Two types of tests were carried out: free desiccation tests and constrained desiccation tests. Free desiccation tests consisted in drying a bar at fixed temperature $\left(18{ }^{\circ} \mathrm{C}\right)$ and relative humidity $(40 \%)$, on a Teflon substrate (which minimises the boundary constraint). Strain/water content data were obtained by caliper measurements and weighing. Water content repartition throughout the sample was also monitored. For constrained desiccation 
876 European Journal of Civil and Environmental Engineering. Volume $13-\mathrm{n}^{\circ} 7-$ $8 / 2009$

tests, a constraint was created at the bottom surface in the axial direction only, by using a substrate with thin parallel lateral notches. In both cases, horizontal strains were measured at mid height; vertical strain was measured in the centre of the bar.

For all of the free desiccation tests, none of the bars experienced cracking. The strain / water content evolution from $w=32 \%$ (liquid limit value) to $20 \%$ (shrinkage limit) was quasi-linear and isotropic. Above the liquid limit, the bar was in a quasi-liquid state, and its behavior is not considered here. Local water content measurements reveal small heterogeneities between bar center and extremities, which disappears below $w=25 \%$.

For all of the constrained desiccation tests, due to the parallel notches, the value of axial strains is $0.5 \%$ at the onset of cracking, hence much lower than in the free desiccation tests at comparable water content. Strains in the transversal direction are also lower (due to a slight friction). In turn, vertical strains are larger. Cracks appeared successively in the bar at an average water content range comprised between 24 and $22 \%$. They formed a parallel set normal to the axial constraint (see Figure 1). Several cracks often formed simultaneously; more commonly they would cut the bar in two pieces, and then the formed pieces again in two pieces. Just before the appearance of the main cracks, small, localised cracks were observed at the bar bottom corner, causing a slight detachment of the bar extremities from the support.

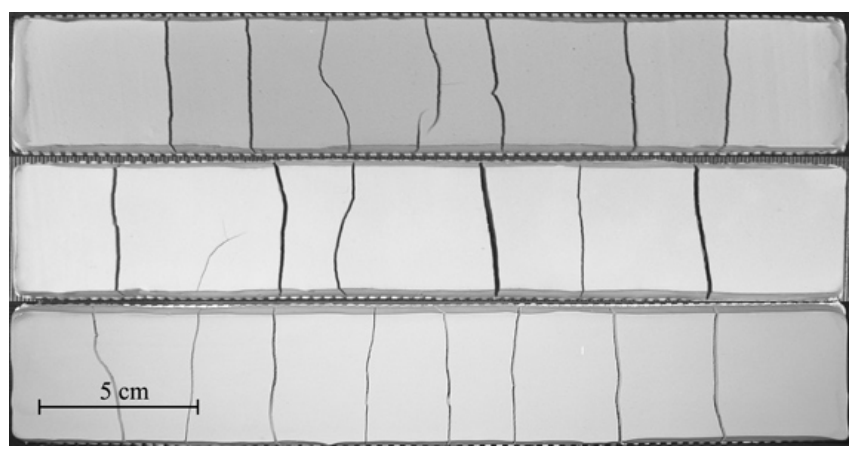

Figure 1. Examples of desiccation crack one dimensional patterns.

\subsubsection{Desiccation tests on slabs}

Desiccation tests were performed, for which a bottom substrate was devised with 2D constraints, instead of the 1D constraint considered so far. Slabs with two different heights (4 $\mathrm{mm}$ and $12 \mathrm{~mm}$ ) were tested. All tested samples cracked, leading to formation of a bi-dimensional net of cracks (see Figure 2). Marked differences were observed between samples that were $4 \mathrm{~mm}$ high and samples that were $12 \mathrm{~mm}$ high. The former ones experienced a large number (from 160 to 352) of relatively thin cracks; the latter ones experienced a small number of relatively wide cracks (from 16 to 31). Corte and Higashi (1960) observed the same trend in drying square shaped slabs. 

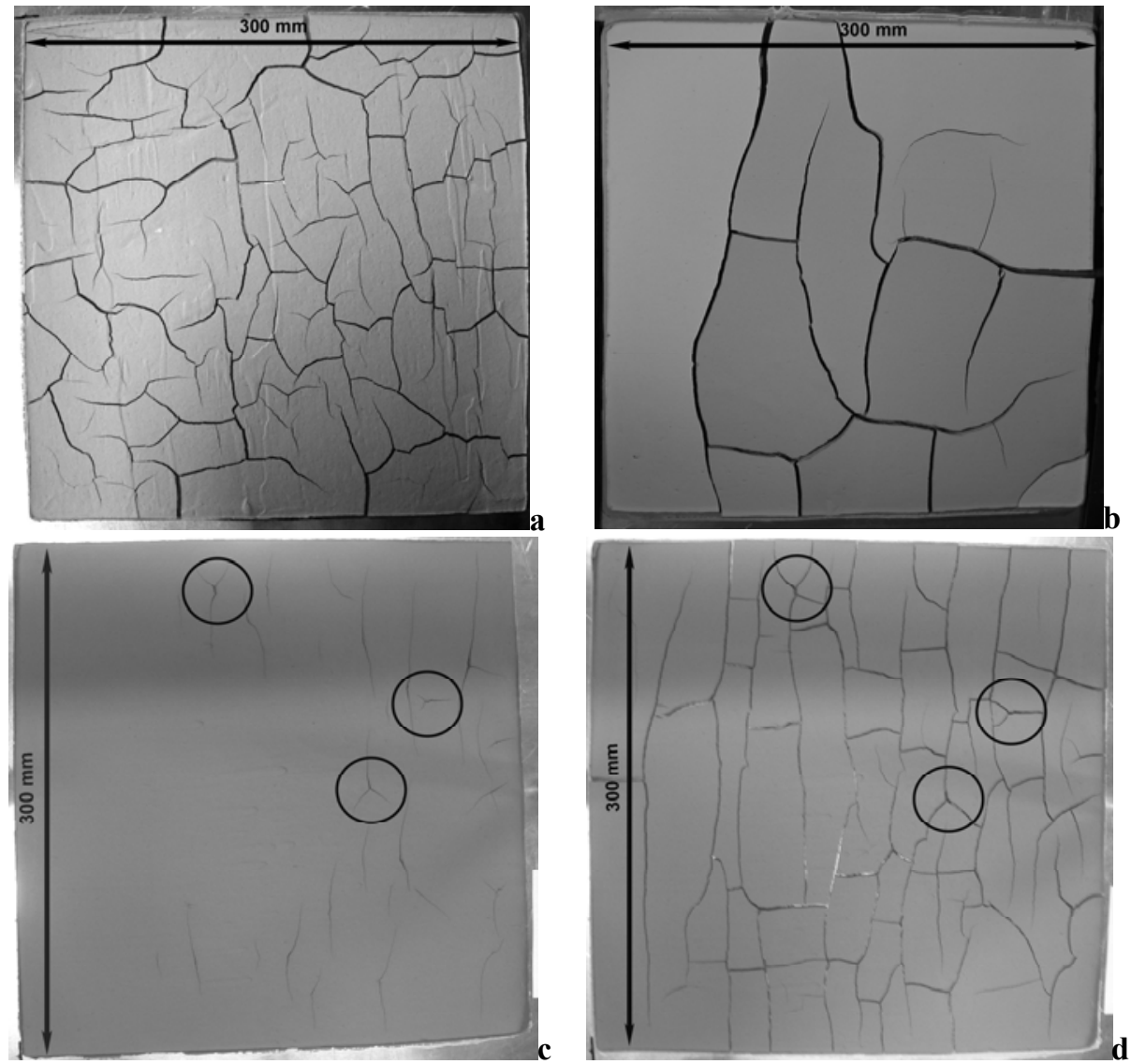

Figure 2. Examples of desiccation crack two dimensional patterns; a, $4 \mathrm{~mm}$ high slab; b, $12 \mathrm{~mm}$ high slab; $c$, initial stage of cracking: $120^{\circ}$ intercepting cracks circled; d, final stage of cracking: $120^{\circ}$ intercepting cracks circled.

Crack intersecting angles were also measured. Generally, cracks intersect at about $90^{\circ}$, up to about $150^{\circ}$. Angles above and beyond this range are rare. During tests with $4 \mathrm{~mm}$ high slabs, it was observed that some groups of three cracks intersecting at $120^{\circ}$ had the tendency to appear simultaneously, especially at the beginning of the cracking stage (Figure 2c, circled cracks). Upon further desiccation, the crack pattern changed, and cracks tended to meet other existing cracks that formed an angle of about $90^{\circ}$ (Figure 2d, photograph taken 4 hours later when all the cracks are formed). It appears that a 2D constraint tends to originate two limiting kinds of crack patterns: $90^{\circ}$ intersecting and $120^{\circ}$ intersecting cracks. It can be deduced that the geometry of the crack pattern strongly depends on the nature of the boundary constraint and the sample geometry. 
878 European Journal of Civil and Environmental Engineering. Volume $13-\mathrm{n}^{\circ} 7$ 8/2009

\subsection{Drying shrinkage}

Apart from the desiccation tests, Water Retention Curve (WRC) was determined for Bioley clayey silt (i.e. the soil used for desiccation tests, see the previous section) as well as two other soils, namely Sion silt and La Frasse clay. The strains and the degree of saturation at different stages of drying and re-wetting were determined (Peron et al. 2007) for different types of soils. Figure 3 features the obtained WRC. It appeared that (Peron et al. 2009):

- (1) Air entry value and shrinkage limit are very close to each other.

- (2) Strains experienced during desiccation up to the air entry value (as long as $\mathrm{S}_{\mathrm{r}}$ remains close to $100 \%$ ) are mainly irreversible; this stage is followed by a domain with lower and mostly reversible deformations at decreasing $S_{\mathrm{r}}$.

- (3) Cracking corresponds to the end of the saturated stage of drying, close to air entry value. This observation was confirmed by tests on other materials (a silt and a silty clay). This behaviour is highlighted in Figure 4 in which the evolution of the degree of saturation is reported as drying proceeds, as well as the moment of cracking initiation, for three different soils. Cracking initiates at degree of saturation values close to $95 \%$.
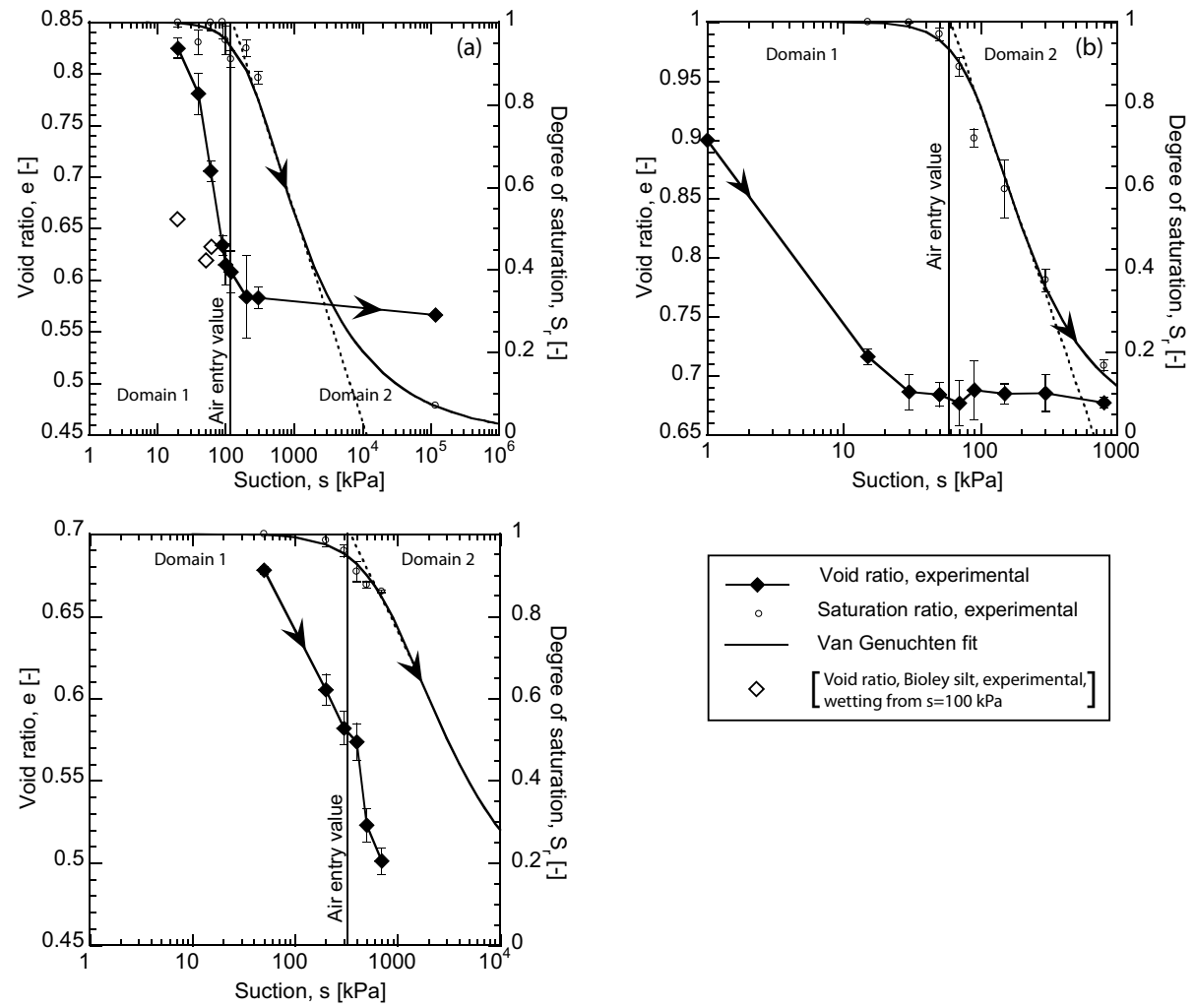

Figure 3. WRC for three soils: (a) Bioley silt, (b) Sion silt, (c) La Frasse clay. 

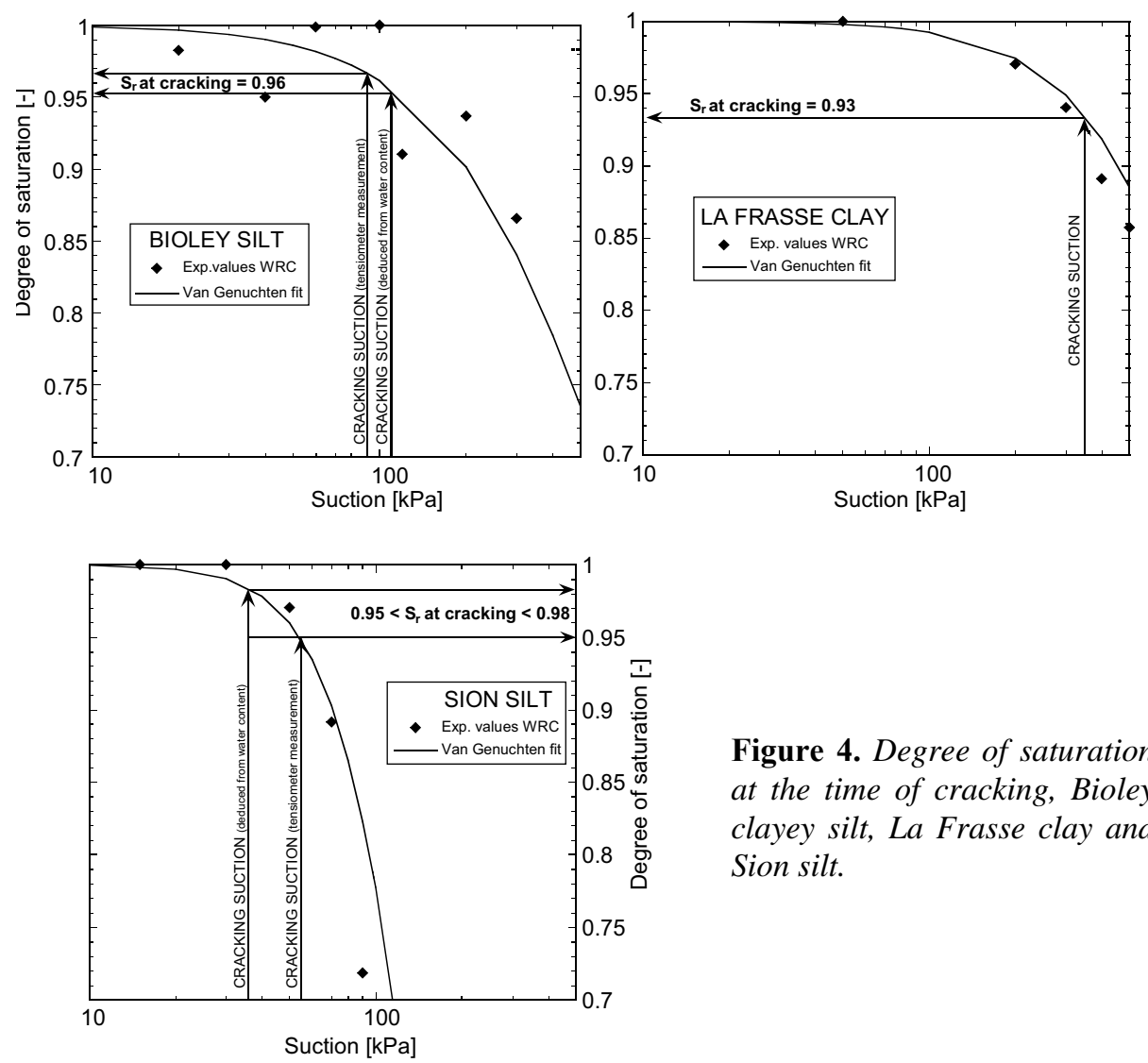

Figure 4. Degree of saturation at the time of cracking, Bioley clayey silt, La Frasse clay and Sion silt.

\section{Desiccation cracking initiation conditions in soils}

Crack initiation conditions are now discussed, starting from the above results regarding water content, suction and degree of saturation at cracking. Some prior results (Corte and Higashi 1960, Ayad et al. 1997, Nahlawi 2004, Avila 2004) are close to the present findings that desiccation cracking of initially saturated and remoulded soils occurs when the overall degree of saturation is $100 \%$. In an attempt to shed light on the role of the air entry in crack initiation in saturation conditions, it may be useful to address phenomena arising during drying at the pore scale. Some studies proposed that defects and air-filled pores may act as crack initiators in soils (Frydman 1967, Snyder and Miller 1985, and Morris et al. 1992).

It may be of interest that drying gels have also demonstrated that cracking has a tendency to occur as the material degree of saturation is still very close to $100 \%$ (within the measurement error range), and its water content is not far from air entry and shrinkage limit (Dwivedi 1986, Simpkins et al. 1989) in laboratory tests. Cracking near the air entry value has been linked to the formation of a drying front 
880 European Journal of Civil and Environmental Engineering. Volume $13-\mathrm{n}^{\circ} 7$ $8 / 2009$

in the gel at the moment of air entry (Scherer 1990). Drained zones with a certain extension, shape and orientation with respect to the stress field could be considered as defects, increasing the probability of crack inception.

Water cavitation may be also a way for dry zones to appear directly inside water-filled pores. Cavitation is observable when the water pressure at a point falls below the water vapour pressure at the local ambient temperature. Water undergoes a phase change, creating vapour-filled voids, termed cavitation bubbles. This situation is likely to occur for a suction value greater than about $100 \mathrm{kPa}$ (atmospheric pressure), since the saturation vapour pressure of water at ambient temperature is very low (approximately $3 \mathrm{kPa}$ ).

The growth of a gas bubble requires overcoming an energy barrier. Or and Tuller (2002) recall that the energy $\Delta E_{B}$ required to form a gas bubble isolated in the liquid phase is the sum of the energy of a newly created interface and the work of the negative pressure over the bubble volume, given by:

$$
\Delta E_{B}=4 \pi r_{c}^{2} T_{s}+\frac{4 \pi}{3} r_{c}^{3}\left(p_{g}-p_{w}\right)
$$

Where $r_{c}$ is the radius of the bubble, $T_{s}$ is the water-vapour surface tension, and $p_{g}$ $p_{w}$ is the difference between air and water pressure, which is often identified as the matric suction.

The maximum of this energy variation is reached for a bubble radius equal to:

$$
r_{c}^{*}=\frac{-2 T_{s}}{p_{g}-p_{w}}
$$

which is the well-known Young-Laplace equation.

In other words, for a given (negative) pressure, bubbles smaller than the critical radius given by Equation [8] do not grow. Above the critical radius, the energy of the system decreases with increasing bubble radius. Thus, cavitation is likely to occur for water pressure below its saturation vapour pressure and only from the bubbles with the critical radius (at a given pressure).

There are two common cavitation circumstances to be distinguished. In case (i), there are no initial gas bubbles that could be acting as cavitation nuclei; hence, water can reach a very high suction value without experiencing cavitation. As tension increases, however, formation of a gas bubble, being energetically favourable, becomes more and more probable. This is homogeneous nucleation. In case (ii), gas bubbles pre-exist (commonly the case). In this situation, cavitation occurs as soon as the water pressure reaches a value corresponding to the critical radius. 
In situation (i), the number of possible nucleations per unit time per unit volume of liquid can be determined using Maxwell-Boltzmann's statistics (Fisher 1948). A rapid calculation indicates that the range of suction $(0-500 \mathrm{kPa})$ for which the material is likely to crack is largely below values that could involve observable homogeneous cavitation. Thus, cavitation in the discussed materials would more probably be the consequence of heterogeneous nucleation. Again, a calculation with Equation [8] indicates that nuclei of about $1.45 \mu \mathrm{m}$ are sufficient to initiate cavitation within water mass at a negative pressure of about $100 \mathrm{kPa}$.

From the cracking onset point of view, in order for a formed bubble to grow out of the pore where it was created, suction in the surrounding water must be great enough to allow the water vapour bubble to pass through the porous network. This condition was discussed by Scherer and Smith (1995) for drying gels, and Or and Tuller (2002) for soils. Thus, suction must be higher than the air entry value. If this is the case, larger unsaturated pockets might be created, acting as flaws and possibly promoting crack initiation, in the same way as discussed above. Such "pockets" may also appear earlier than the overall air entry in a given sample, depending on the existence of isolated larger pores that could be drained early in the process.

It is noted that the direct application of the Young-Laplace law to the cavitation phenomenon has been found in many technical circumstances to be misdirected (see e.g., Brennen 1995). Particularly for soil pore water, heterogeneous cavitation is thought to occur at the solid walls of pores, with the radius of cavity primarily determined by the pore geometry.

In conclusion, the air entry value (or better said the gas entry value) can be associated with the formation of localised drained zones, either by the cavitation near the existing inter-phase boundary by generating water vapour, which subsequently merges with the external gas environment, or cavitation deep within the body followed by a migration or growth of the air bubbles, which subsequently act as material defects. These defects can then become crack inceptors. The latter scenario is related to the observation that locally, the moment of gas entry is critical for crack initiation in the discussed test conditions. However, the present tests do not give direct evidence of the effectiveness of such a mechanism.

The presence of defects must be invoked; a crack starts from some kind of inceptor. Macroscopically, tensile strength could be viewed as a property related to such defects; similarly, suction and degree of saturation changes are also defectrelated properties.

Based on the actual porosimetry data for Bioley silt and Sion silt (Peron 2008), a micro-scale hydro-mechanical model has been proposed to simulate the shrinkage deformation and subsequent cavitation occurring in different classes of pores (see $\mathrm{Hu}$ et al 2008, 2009). The model exploits an earlier idea of Or and Tuller (2002), according to which the size of a cavitation created bubble is equal to the size of the soil pore. For a bimodal pore model, it was found that water in larger pores cavitates much earlier than in smaller pores. An averaged suction-saturation-deformation 
882 European Journal of Civil and Environmental Engineering. Volume $13-\mathrm{n}^{\circ} 7$ $8 / 2009$

pattern seen in macroscopic experiments has been reproduced by simulating a twopore system (Hu et al. 2008).

\section{Scenarios of desiccation crack pattern formation}

\subsection{Interpretation of desiccation of bars (one dimension case)}

So as to further quantify such a process, a stress field arising from the desiccation of the bar (section 3.1.1) has been modelled via finite elements. Strains are here viewed as a combination of a drying shrinkage induced (volumetric) part $\varepsilon^{h}$ proportional to water content change $\Delta w$, and a mechanical part generated to satisfy strain compatibility (which induces stresses). Then, the analogy between the equation describing moisture transport and the elastic response to changes in water content, on the one hand, and the thermo-elasticity of the heat diffusing elastic medium, on the other hand has been used. Note again that this is a single-phase approach. No pore water pressure and/or effective stress effects are addressed. The elastic formulation that is used here produces a valid stress field (Peron et al., 2009), but one has to be aware that most of the deformations produced during the first drying of a soil mass are irreversible as seen in section 3.3.

The field and constitutive equations of the numerical model are hereafter summarized. The linear momentum conservation law reads:

$$
\operatorname{div} \boldsymbol{\sigma}+\rho \mathbf{g}=0
$$

where $\sigma$ is the total (Cauchy) stress tensor with tensile stresses taken as positive. $\rho$ is the total mass density of the medium.

In what follows we limit our considerations to the linear theories. The mechanical behaviour of the whole medium is assumed to be governed by total stress, which is related to a mechanical elastic strain $\varepsilon^{\mathbf{m e}}$ through:

$$
\boldsymbol{\sigma}=\mathbf{D}: \varepsilon^{\mathrm{me}}
$$

where $\mathbf{D}$ is the linear elastic constitutive tensor.

Assuming that drying induced water mass transfer through the body is solely governed by a diffusion law and neglecting the deformation influence on water mass transfer (Peron 2008), mass conservation of water states:

$$
\partial_{t} w-\operatorname{div}[\lambda \operatorname{grad} w]=0
$$

where $\lambda$ is a diffusivity coefficient $\left[\mathrm{m}^{2} . \mathrm{s}^{-1}\right]$, and $w$ is the gravimetric water content. 
The total strain (positive in compression) is divided into two parts, the drying induced part due to water mass removal and the mechanical part due to total stress variation. The mechanical strain rate is assumed to be purely elastic and the drying induced strain rate is assumed to be proportional and opposite in sign to water content increment $d w$. These assumptions lead to:

$$
d \varepsilon_{v}=\frac{d p}{K}+\alpha \cdot d w ; \quad d \varepsilon_{d}=\frac{d q}{3 G}
$$

where $K$ and $G$ are bulk and shear elastic moduli. $d \varepsilon_{v}$ and $d \varepsilon_{d}$ are volumetric and deviatoric strain rate invariants, $\mathrm{p}$ and $\mathrm{q}$ are mean and deviatoric stress invariants. $\alpha$ is a hydric expansion/shrinkage coefficient.

Considering the boundary conditions, the weak form of the governing equations may be obtained through a variational approach. The governing equations written in matrix form become:

$$
\left[\begin{array}{cc}
\mathbf{K} & 0 \\
0 & \mathbf{H}_{1}
\end{array}\right]\left\{\begin{array}{l}
\overline{\mathbf{u}} \\
\bar{w}
\end{array}\right\}+\left[\begin{array}{cc}
0 & 0 \\
0 & \mathbf{H}_{2}
\end{array}\right]\left\{\begin{array}{c}
0 \\
\partial_{t} \bar{w}
\end{array}\right\}=\left\{\begin{array}{c}
\mathbf{f}_{\mathbf{u}} \\
\mathbf{f}_{w}
\end{array}\right\}
$$

where $\overline{\mathbf{u}}$ and $\bar{w}$ are the nodal unknowns, $K$ is the stiffness matrix, $\mathbf{H}_{\mathbf{1}}$ is a "water diffusivity" matrix and $\mathbf{H}_{2}$ is a time dependence matrix. $\mathbf{f}_{\mathbf{u}}$ and $\mathbf{f}_{\mathbf{w}}$ are nodal flux vectors.

The formulation of the problem is analogous to thermal diffusion in an elastic body. It is solved as follows. First the water content field is determined. Then water content field intervenes as a solicitation for the determination of the displacement field. Eventually, in Equation [13], the term $\mathbf{f}_{\mathbf{u}}$ includes a nodal force term due to the drying induced strain, deduced from the water content.

A 2D model of the bar used in desiccation tests has been examined. The bar (under plain strain hypothesis) has been subjected to a condition of zero displacement at the bottom. Drying boundary conditions has consisted in imposing decreasing water content values on the surfaces of the top and side extremities of the bar, with a constant rate of $1.2 \%$ per hour (as recorded during the experiments). The simulation has been performed with the finite element code GefDyn (Modaressi et al. 1991; Aubry et al., 1995). Linear elements are used for the computation of water content, and quadratic elements for the displacements computation. An implicit time integration scheme is adopted. The extensive results can be found in $\mathrm{Hu}$ et al. (2006), Peron et al. (2008); only the main results are summarized here, regarding crack initiation location.

Results (Figure 5) show that maximum tensile stress (in absolute value) in horizontal direction is reached along the central vertical section (although the minor stress is almost constant in a large central part of the bar), indicating the first vertical 
884 European Journal of Civil and Environmental Engineering. Volume $13-\mathrm{n}^{\circ} 7-$ $8 / 2009$

crack would initiate at this location. In the conditions of the simulation, fracture mechanics theory shows that such a crack is unstable (Bai et al., 2000), and should fully propagate through the specimen thickness. The cracking process could be resumed in the new sample pieces resulting from the first vertical cracking, leading to the formation of regular crack spacing. As a deviation from this scheme, it is noted here that many times, cracks appeared simultaneously within the bar.

The slight detachment experimentally observed near the bottom extremity is explained by a shear effect. It induces large but concentrated tensile stresses, generating conditions for early crack formation. The complex stress field in this zone certainly prevents from larger crack extension.

a
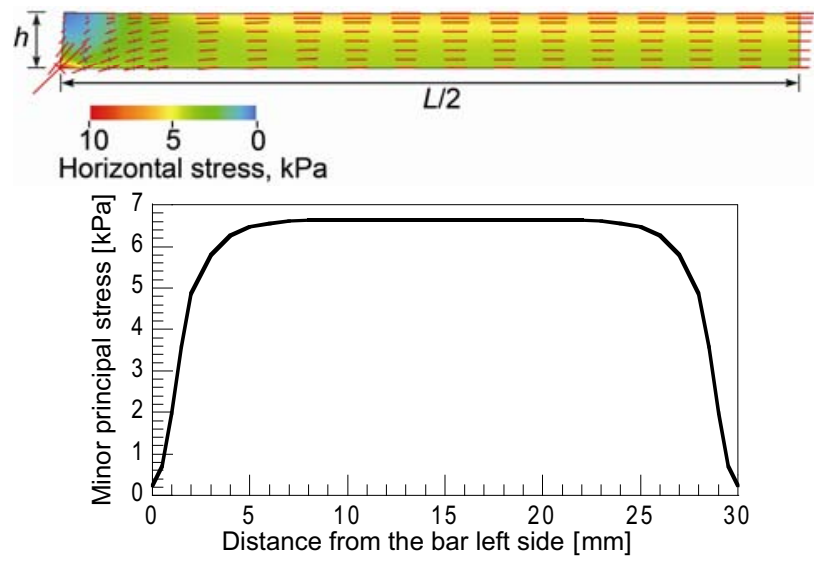

Figure 5. Simulation of constrained desiccation tests (tensile stresses positive). a, minor principal stress and horizontal stress fields (half bar), onset of cracking (red lines, tensile stresses, blue lines, compressive stresses). b, minor principal stress along the bar top surface.

\subsection{Interpretation of desiccation of slabs (two dimension case)}

The formation of the two dimensional crack patterns observed in section 3.1.2 is now addressed. The interpretation $90^{\circ}$ crack intersection angle is straightforward. Once a crack has formed, tensile stresses perpendicular to the crack axis are released near the crack. A propagating crack, when approaching the vicinity of another existing crack, tends to orientate in the direction perpendicular to the local maximum tensile stress, i.e. parallel to the existing crack.

The case of $120^{\circ}$ intersection angles results from an energy minimisation process, as demonstrated for soils earlier, and reported by Corte and Higashi (1960). First, cracking, by creating new solid surfaces, is an energy consuming process. Consider an infinite slab subjected to a horizontal two-dimensional constraint while it dries. If the tensile stress at a given point is isotropic, then the sample tends to crack along several planes of failure. Of the infinite theoretical possibilities, failure 
will occur in three planes oriented at $120^{\circ}$, because this geometry produces the lowest crack area enclosing the maximum soil volume and thus enables the consumption of a minimum surface energy per unit volume. This reflects the tendency of any physical process to do the most with the least.

The interpretation provided in section 5.1 for one dimensional crack patterns can be extended to the two dimensional case in order to interpret the ensuing patterns with regular crack spacing also observed in this case.

\subsection{Further considerations}

With interpretation similar to the one of section 5.1, Groisman and Kaplan (1994) proposed to relate crack spacing to dried slab height. Desiccation of circular slabs was considered, whose shrinkage was restrained in the horizontal plane because of friction between the soil and its bottom support. The process of fragmentation of the specimen basically consists in the successive cracking of individual cells between adjacent cracks, where the stress reaches the tensile strength. The process must stop when the dimension of the cells drops below a critical value, as the bottom friction ceases to supply the stress needed for cracking. Such a critical value directly depends on the height of the cell.

In conclusion, if one assumes that cracks form successively through incremental growth to the material tensile strength in locations well defined by the stress field and the boundary conditions, it is relatively easy to interpret the formation of the crack pattern. Final crack spacing should be the consequence of changes in tensile strength or other material properties. When the crack pattern emerges from a homogeneous stress field, it is less straightforward how to quantify the observed process. Fracture mechanics should be invoked, i.e., global energy considerations. Derivation of crack spacing using such an approach can be found in Peron et al. (2009), as well as in Bazant and Cedolin (1991).

\section{Conclusion}

Desiccation can be seen as a set of processes that include drying, shrinkage and cracking. For a relatively high saturation ratio and continuous liquid phase, the basic process behind the shrinkage of a soil is a liquid pressure decrease / suction increase, caused by evaporation at the level of menisci, which acts as an attractive force between the components of the matrix. Therefore, the matrix suffers an increasing internal compression and the sample shrinks. Experimental evidence clearly shows that desiccation cracking occurs in mode I (i.e. opening mode), indicating that cracking initiation is the result of soil tensile strength mobilization. The factors controlling shrinkage and cracking were examined. Based on this analysis, the following conclusion can be drawn: 
886 European Journal of Civil and Environmental Engineering. Volume $13-\mathrm{n}^{\circ} 7$ $8 / 2009$

1. Free desiccation tests on initially saturated slurries confirm the general characteristics well known from WRC studies, i.e., the existence of two distinct domains for drying shrinkage (Domain 1 at a degree of saturation close to one, with mostly irreversible deformation, and Domain 2 at a decreasing degree of saturation, with a much smaller deformation, mainly reversible).

2. The experimental results presented in this paper show that desiccation cracking occurs in Domain 1 at non-zero suction and a degree of saturation almost equal to $100 \%$, close to the onset of air entry. The increase of suction in the drying body alters the liquid phase repartition (degree of saturation) and occupation of the porous space; this possibly modifies the conditions for desiccation crack initiation during drying. Furthermore, this coincidence could be related to the fact that tensile strength evolves during drying. Indeed it is known that the increase of suction induces an increase of the apparent cohesion.

3. The stresses that lead to desiccation cracking clearly result from the presence of restraining boundary conditions and/or moisture gradients. During this stage, a large part of deformation is irreversible. Stress builds up until a critical point at which the tensile strength is met. The crack patterns may be interpreted by examining the stress field at that time. Regular crack pattern geometry then stems from a recursive process of tensile strength mobilization which ends once the conditions for crack initiation (or propagation) cannot be reached anymore

\section{References}

Abu Hejleh A., Desiccation theory for soft cohesive soils, PhD. Thesis, University of Colorado, Boulder, 1993.

Albrecht B.A, Benson C.H., "Effect of desiccation on compacted natural clay”, J. Geotech. Geoenviron., vol. 127, $\mathrm{n}^{0} 1$, 2001, p. 67-75.

Aubry D., Chouvet D., Modaressi A., Modaressi, H., Gefdyn: logiciel d'analyse du comportement mécanique des sols par éléments finis avec prise en compte du couplage sol-eau-air, Report, 1995, Ecole Centrale Paris.

Avilá G., Estudio de la retracción y el agritamiento de arcillas - Aplicación a la arcilla de Bogota, PhD. Thesis, Universitat Politecnica de Catalunya, Barcelona, 2004.

Baggio P., Bonacina C., Schrefler, B.A., "Some considerations on modelling heat and mass transfer in porous media”, Transport Porous Med., vol. 8, 1997, p. 233-251.

Bai T., Pollard D.D., Gao H., Explanation for fracture spacing in layered materials. Nature, vol. 403, 2000, p. 753-756.

Bazant Z.P., Cedolin L., Stability of Structures - Elastic, Inelastic, Fracture, and Damage Theories, Oxford University Press, 1991.

Bishop A., Garga, V., “Drained tension tests on London Clay”, Géotechnique, vol. 19, 1969, p. 309-313. 
Brennen C.E., Cavitation and Bubble Dynamics, Oxford University Press, 1995.

Corte A., Higashi A. Experimental Research on Desiccation Cracks in Soil, Research report 66, 1960, U.S. Army Snow and Ice and Permafrost Research Establishment.

Coussy O. Eymard R., Lassabatère T., Constitutive modelling of unsaturated drying deformable media, J. Eng. Mech. ASCE, vol. 124, nº6, 1998, p. 658-667.

Daniel D., Wu, Y., “Compacted clay liners and covers for arid sites”, J. Geotech. Eng. ASCE, vol. $119 \mathrm{n}^{0} 2$, 1993, p. 223-237.

Dwivedi K., “Drying behavior of alumina gels”, J. Mater. Sci. Lett., vol. 5, 1986, p. 373-376.

Fisher R.A., "On the capillary forces in an ideal soil: Correction of the formulae given by W.B. Haines”, J. Agr. Sci., vol. 16, 1926, p. 492-503.

Frydman S., "Triaxial and tensile strength tests on stabilized soil”, Proceedings of the third Asian Regional Conference on Soil Mechanics and Foundation Engineering, Haifa, 1967, p. $269-275$.

Griffith A.A., "Theory of rupture”, Proceedings of the First International Conference on Applied Mechanics, Delft, 1924, p. 55-63.

Groisman A., Kaplan, E., “An experimental study of cracking induced by desiccation”, Europhys. Lett., vol. 25, nº, 1994, p. 415-420.

Hu L., Peron H., Hueckel T., Laloui L., "Numerical and phenomenological study of desiccation of soil”, Advances in Unsaturated Soil, Seepage, and Environmental Geotechnics, ASCE Geotechnical Special Publication 148, 2006, p. 166-173.

Hu L., Hueckel T., Peron H., Laloui L., “Modeling evaporation, shrinkage and cracking of desiccating soils”, $12^{\text {th }}$ International Conference of International Association for Computer Methods and Advances in Geomechanics (IACMAG), 2008, p. 1083-1090.

Hu L., Hueckel T., Peron H., Laloui L., "Shrinkage of desiccating soils: a bi-modal porosity model”, Water Resour. Res., 2009, submitted.

Hueckel T., "On effective stress concept and deformation in clays subjected to environmental loads: Discussion”, Canadian Geotechnical Journal, vol. 29, 1992, p. 1120-1125.

Kim T., Hwang C., "Modelling of tensile strength on moist granular earth material at low water content”, Engineering Geology, vol. 69, 2003, p. 233-244.

Kleppe J.H., Olson, R.E., “Desiccation cracking of soil barrier”, Hydraulic Barrier in Soil and Rock, ASTM STP 874, 1985, p. 263-275.

Konrad J.M., Ayad R., “An idealized framework for the analysis of cohesive soils undergoing desiccation”, Can. Geotech. J., vol. 34, 1997, p. 477-488.

Kowalski S.J., Thermomechanics of Drying Processes, Springer Verlag, 2003.

Lachenbruch A.H., „Depth and spacing of tension cracks”, Journal of Geophysical Research, vol. 66, n ${ }^{0} 12$, 1961, p. 4273-4292.

Nuth M, Laloui L., "Effective stress concept in unsaturated soils: Clarification and validation of a unified framework”, Int. J. Numer. Anal. Met. Geomech., vol. 32, 2008, p. 771-801. 
888 European Journal of Civil and Environmental Engineering. Volume $13-\mathrm{n}^{\circ} 7$ 8/2009

Mainguy M., Coussy O., Baroghel-Bouny, V., "Role of air pressure in drying of weakly permeable materials”, J. Eng. Mech. ASCE, vol. 127, n6, 2001, p. 582-592.

Mitchell, J.K., Soga, K., Fundamentals of Soil Behavior, John Wiley \& Sons, 2005.

Modaressi H., Laloui L., Aubry D., “Numerical modelling of thermal consolidation”, Second European Conference in Numerical Methods in Geotechnical Engineering, Santander 90, 1991, p. 280-292.

Morris P.H., Graham, J., Williams, D.J., “Cracking in drying soils”, Can. Geotech. J., vol. 29, 1992, p. 262-277.

Nahlawi H., Behaviour of a reactive soil during desiccation, Master Thesis, Monash University, Clayton, Australia, 2004.

Or D., Tuller M., “Cavitation during desaturation of porous media under tension”, Water Resour. Res., vol. 38, n5, 2002, 19-1-19-4.

Peron H., Desiccation Cracking of Soils, PhD. Thesis, Ecole Polytechnique Fédérale de Lausanne, 2008.

Peron H., Hu L., Laloui L., Hueckel T., "Mechanisms of desiccation cracking of soil: validation", International Symposium on Numerical Models in Geomechanics - NUMOG X, 2007, Taylor \& Francis Group, p. 277-282.

Peron H., Hueckel T. Laloui L., “An improved volume measurement for determining soil water retention curve”, Geotech. Test. J., vol. 30, nº1, 2007, p. 1-8.

Peron H., Delenne J.Y., Laloui L., El Youssoufi M.S., "Discrete element modelling of drying shrinkage and cracking of soils”, Computers and Geotechnics, vol. 36, 2009, p. 61-69.

Peron H., Hueckel T., Laloui L., Hu L., "Fundamentals of desiccation cracking of finegrained soils: experimental characterisation and mechanisms identification”, Can. Geotech. J., 2009, In Press.

Philip J.R., de Vries D.A., "Moisture movement in porous materials under temperature gradients”, Transactions of the American Geophysical Union, vol. 38, 1957, p. 222-232.

Platten J.K., “The Soret effect: a review of recent experimental results”, J. Appl. Mech. T. ASME, vol. 73, 2006, p. 5-15.

Scherer G.W., “Theory of drying”, J. Am. Ceram. Soc., vol. 73, nº1, 1990, p. 3-14.

Scherer G.W., Smith D.M., „Cavitation during drying of a gel”, J. Non-Cryst. Solids, vol. 189, 1995, p. 197-211.

Schubert K., Kapillarität in porösen Feststoffsystemen, Springer Verlag, 1982.

Snyder V.A., Miller R.D., “Tensile strength of unsaturated soils”, Soil Sci. Soc. Am. J., vol. 49, 1985, p. 58-65.

Simpkins G., Johnson Jr. D.W., Fleming D.A., “Drying behavior of colloidal silica gels”, $J$. Am. Ceram. Soc., vol. 72, nº10, 1989, p. 1816-1821. 